\title{
Simplified Computation of The Heat Transfer Co- efficient in Quenching
}

\section{Imre Felde}

John von Neumann Faculty of Informatics, Óbuda University, Budapest, Hungary, felde@uni-obuda.hu

\begin{abstract}
Due to the direct observation of the Heat Transfer Coefficient at the surface of the metallic components under quenching is practically impossible, indirect methods based on measuring the cooling curves in certain points inside the workpieces, and numerical integration of their thermodynamic model mean a viable approach. The complexity of the necessary calculations can be considerably reduced by the use of symmetric cylindrical samples made of an alloy of particularly simple thermal properties defined in the standard ISO 9950. In spite of that the complexity is still large enough. In the present approach it is reduced by applying a simple formal, qualitative model of the cooling process, the efficient Newton-Raphson algorithm and a Fixed Point Iteration approach to obtain approximate preliminary results. This approach requires only very limited computational capacities. Besides for making rough estimations, due to its simplicity, the method may be useful in the education.
\end{abstract}

Keywords: Heat Transfer Coefficient; Newton-Raphson Algorithm; Banach's Fixed Point Theorem; Fixed Point Iteration; Quenching

\section{Introduction}

In many technical applications rigorous deduction of the results on the basis of the available physical models and fundamental principles is impossible due to the high complexity of these tasks. In quenching of metallic components this complexity often roots in the complicated initial and boundary conditions, the turbulent flow of the cooling fluid, phase transitions in the fluid as condensation, evaporation that brings about heat insulating gas bubbles at the surface of the quenched components. Phase transitions and simultaneous chemical reactions within the solid sample may serve as heat sources or sinks, etc. To partly reduce these complexities, in the standard ISO 9950 [1] a cylindrical sample of well defined shape, size, and material composition is applied with internal points in which the temperature can be measured by thermocouples. The task can be formulated as the determination of the "Heat Transfer Coefficient" (HTC) on the surface of the sample as the function of the time, in the possession of the measured cooling curves, the heat conduction model, and the main thermodynamic data of the component that are available for some key 
engineering alloys [2]. If this task is tackled by assuming explicit $H T C$-time functions over the surface of the sample, finite element mathematical approximation and numerical integration of the equations becomes possible to determine the cooling curves. The so computed curves can be compared with the measured ones, and by modification of the original assumption on the HTC, efforts can be done to reduce these differences (e.g. [3]).

Even if the cylindrical symmetry of the probe defined in [1] is taken into account, the solution of this "Inverse Heat Transfer Problem" needs great computational power. For instance in [4] gradient-based and genetic algorithm was applied with complementary utilization of the graphical card's computational capacities of the computers.

As an alternative of using high computational power in technical applications the systematic utilization of certain quantitative numerical values and some available qualitative modeling knowledge can be mentioned that at first obtained rigorous mathematical framework in the theory of fuzzy sets [5]. By the combination of the qualitative and quantitative information simple functions with appropriate "shape parameters" can be defined, and these parameters can be fitted to the measured data for obtaining good numerical approximations. In Dombi's "Pliant Systems" introduced in [6] the so-called "distending function" is used due to which the various operators are closely related to each other by setting certain form parameters. Similarly, "computationally cheap approximation" of important probability distribution functions (the normal, epsilon, omega), and the kappa regression function by typical functions of certain form parameters can be found (e.g. [7]). Similar situation can be observed in modeling dynamical phenomena having long memory properties: they can be described only by very high order differential equations containing numerous parameters. However, by using fractional order models (the brief history of the topic is given in [8]) instead of the integer order ones, only a few free parameters can be well fitted to the practical problems.

In the present paper a similar approach is outlined by observing the qualitative formal properties of the cooling curves and the calculated HTC vs. time functions given in [9].

For the approximation of these structures it is assumed that at the surface of the sample the function $H T C$ vs. time can be expressed as $H T C \equiv h(T(\mathbf{r}, t))$ (variable $t[s]$ means the time, vector $\mathbf{r}$ denotes the location of the point on the surface of the probe, and $T[C]$ is the temperature). For the single variable $h(T)$ functions various formal suggestions are given. Assuming that the length of the probe is long enough for making the problem symmetric to its centreplane at the half length, the heat transfer in the longitudinal direction must be zero. Furthermore, by utilizing the cylindrical symmetry of the probe, the simplified distribution function $T(r, t)$ was considered, in which $r[m]$ denotes the radial distance from the centreline. The appropriate "format functions", the finite element model and the Euler-integration applied, as well as the methods used for tuning the parameters are discussed in Section 2. 


\section{Formulation of the Problem for "FEM" Approach}

For obtaining curves similar to that given in 2 simple qualitative physical considerations were done. Quenching of metal products is a complex process that is difficult to precisely describe by physical models. The HTC at the surface of the component is a parameter that may depend on the nature of the bulk flow (laminar or turbulent), the temperature, density, viscosity, the latent heat of evaporation/condensation, and other thermal properties of the cooling liquid, the surface quality, shape, and thermal data of the component under quenching. It is mainly determined by physical processes as follows: i) Even if the "bulk" of the cooling liquid has turbulent flow that allows very efficient heat transfer due to "stirring" the fluid layers, at the boundary layer of the quenched sample laminar flow is formed since the fluid sticks on the surface of the probe. The thickness of this layer is determined by the quality of the surface of the probe, the density and viscosity of the liquid. In this layer the heat transfer process mainly is realized by heat conduction, so it is not very efficient. ii) The viscosity of a liquid normally decreases with increasing temperature, therefore at higher temperatures thinner films with better heat transfer abilities are expected. iii) When the temperature achieves the boiling point of the liquid at the pressure of the operation, at the surface of the probe gas bubbles appear that act as "heat insulators", so at higher temperatures some decrease in the HTC value is expected. This effect clearly can be observed optically in the case of refrigerators where it is a practical experimental possibility to manufacture the pipes of transparent glass (e.g. [10]). Though the temperature ranges are considerably different, the main physical processes essentially are the same. It can be commonly observed that boiling liquid drops can have relatively long persistence on hot metallic surfaces. iv) When the hot probe is immersed into the cooling liquid, this liquid comes into boiling nearby its surface. Though the latent heat of boiling used to be considerable, the heat insulation made by the gas bubbles normally has more significant effect on the HTC, consequently, in the beginning low $H T C$ values can be expected.

The above argumentation well explains the shape of the curves given in [9]. On this reason simple form functions that potentially are able to model the "asymmetries" in $h(T)$ were investigated as given in (1) in which the parameters $h_{\max }, w, T_{\max }, w_{\text {left }}$, and $w_{\text {right }}$ individually must be set to produce values of order of magnitude represented in [9]. In (1a) the formal asymmetry was taken from Planck's radiation law using the frequency as independent variable. (Certain modification was introduced to eliminate the numerically inconvenient behaviour of the original formula at $T=0$ that may disturb the numerical calculations.) In the other formulae the location of the "centre of asymmetry" is determined by the parameter $T_{\max }$, and its extent depends on the "width parameters" $w_{\text {left }}$ for the lower, and $w_{\text {right }}$ for the higher temperatures. The parameters given in Table 1 were set by the method of "generate and test".

Due to the cylindrical symmetry of the problem the use of polar coordinates was expedient that yields the heat conduction equation for the alloy Inconel 600 as given in (2) in which $t[s]$ denotes the time, $r[m]$ denotes the radius from the centerline as the "independent variables" of the problem, $T[C]$ is the temperature, and it is "formally" taken into consideration, that according to the numerical data published on the thermal properties of this metal in [2], within the temperature range $T \in$ 
$[27,796.45]\left[{ }^{\circ} \mathrm{C}\right]$ no observable "source" or "sink" terms appear.

$$
\begin{aligned}
& h(T)=\frac{h_{\text {max }} T^{3}}{\exp (T / w)-1} \text { modified as } h(T)=\frac{h_{\text {max }} T^{3}}{\exp (D+|T / w|)-1} \\
& h(T)=h_{\max }\left\{\begin{array}{l}
\exp \left(-\left(\left[T-T_{\text {max }}\right] / w_{\text {left }}\right)^{2}\right) \quad \text { if } T \leq T_{\max } \\
\exp \left(-\left(\left[T-T_{\text {max }}\right] / w_{\text {right }}\right)^{2}\right) \quad \text { if } T>T_{\text {max }}
\end{array}\right. \\
& h(T)=h_{\max } \begin{cases}\frac{D}{D+\left(\left[T-T_{\max }\right] / w_{\text {left }}\right)^{2}} & \text { if } T \leq T_{\max } \\
\frac{D}{D+\left(\left[T-T_{\text {max }}\right] / w_{\text {right }}\right)^{2}} & \text { if } T>T_{\max }\end{cases} \\
& h(T)=h_{\max } \begin{cases}\frac{w_{\text {left }}}{w_{\text {left }}+\left[T-T_{\text {max }}\right]^{2}} & \text { if } T \leq T_{\text {max }} \\
\frac{w_{\text {right }}}{w_{\text {right }}+\left[T-T_{\text {max }}\right]^{2}} & \text { if } T>T_{\max }\end{cases}
\end{aligned}
$$

Table 1

The variable parameters in (1) and their "target" values; in (1a) and (1c) $D=7.5 \times 10^{-2}$ was fixed

\begin{tabular}{|l|c|c|c|c|}
\hline Formula & $h_{\max }\left[\frac{J}{s \cdot m^{2} \cdot K}\right]$ & $T_{\max }[K]$ & $w_{\text {left }}[K]$ & $w_{\text {right }}[K]$ \\
\hline \hline$(1 \mathrm{a})$ & 0.0035 & - & $w=89.5$ & - \\
\hline$(1 \mathrm{~b})$ & 5700.0 & 680.0 & 260.0 & 80.0 \\
\hline$(1 \mathrm{c})$ & 6000.0 & 680.0 & 350.0 & 100.0 \\
\hline$(1 \mathrm{~d})$ & 10000.0 & 680.0 & 3500.0 & 1000.0 \\
\hline
\end{tabular}

$\frac{\partial}{\partial r}\left(k(T(r, t)) \frac{\partial T(r, t)}{\partial r}\right)+\frac{k(T(r, t))}{r} \frac{\partial T(r, t)}{\partial r}=\rho C_{p}(T(r, t)) \frac{\partial T(r, t)}{\partial t}$

Under the normal environmental pressure and the given temperature range the density of the alloy is constant $\rho=8420\left[\mathrm{~kg} \cdot \mathrm{m}^{-3}\right]$, while the heat conductivity-temperature function $k(T)\left[J \cdot s^{-1} \cdot m^{-1} \cdot K^{-1}\right]$ can be well described by a third order polynomial fitted to the tabulated data found in [2]. The same holds for the specific heattemperature function $C_{p}(T)\left[\mathrm{J} \cdot \mathrm{kg}^{-1} \cdot \mathrm{K}^{-1}\right]$. The approximations applied are given in (3).

$k(T)=\sum_{\ell=0}^{3} a_{\ell} \cdot(T / 100)^{\ell}, \quad \rho C_{p}(T)=\sum_{\ell=0}^{3} b_{\ell} \cdot(T / 100)^{\ell}$

with $a_{0}=14.398632059, a_{1}=1.479338274, a_{2}=0.0206104081, a_{3}=-0.0006946666, b_{0}=$ $3660692.67678371, b_{1}=466878.975781679, b_{2}=-120161.302924857$, and $b_{3}=11898.306953622$.

Since the location of the temperature sensors in the standard ISO 9950 corresponds to $r=0$ where (2) is singular, for developing "Finite Elements Method (FEM)" for 
computing the $H T C$ and the cooling curves in the calculations the following approximations were done. For the probe of radius $R=6.25[\mathrm{~mm}]$, instead of the exact range $[0, R]$ the practically computable range $[\Delta r, R]$ was so considered that the $[0, R]$ interval was divided into $N \in \mathbb{N}$ equally long subintervals as $\Delta r=\frac{R}{N}$, and in the role of the centre line $r=\Delta r$ was placed. For the grid points $\left\{r_{i} \mid i=2, \ldots, N-1\right\}$ the central estimation of the gradient was used as in (4a). In this manner it was possible to calculate the $2^{\text {nd }}$ term at the LHS of (2). Again, by the application of the central differences, the calculation of the $1^{\text {st }}$ term at the LHS of (2) was possible only for the points $\left\{r_{i} \mid i=3, \ldots, N-2\right\}$ in (4b). The temperature in the centreline was estimated according to (4c). This scheme allowed the estimation of $\partial T / \partial t$ for the same grid-points, and on this basis, the application of a simple Euler-integration according to the time as $T\left(r_{i}, t+\delta t\right) \approx T\left(r_{i}, t\right)+\delta t \frac{\partial T\left(r_{i}, t\right)}{\partial t}$.

$\nabla T\left(r_{i}, t\right) \equiv \frac{\partial T\left(r_{i}, t\right)}{\partial r_{i}} \approx \frac{T\left(r_{i+1}, t\right)-T\left(r_{i-1}, t\right)}{r_{i+1}-r_{i-1}}$, $i \in\{2, \ldots, N-1\}$

$\frac{\partial}{\partial r}\left(k \frac{\partial T}{\partial r}\right) \approx \frac{k\left(r_{i+1}, t\right) \nabla T\left(r_{i+1}, t\right)-k\left(r_{i+1}, t\right) \nabla T\left(r_{i-1}, t\right)}{r_{i+1}-r_{i-1}}$, $i \in\{3, \ldots, N-2\}$

$T\left(r_{1}, t\right) \equiv T\left(r_{2}, t\right) \equiv T\left(r_{3}, t\right)$

The boundary conditions were set by (5)

$-k(T(R, t)) \frac{\partial T(R, t)}{\partial r}=h(t)\left(T(R, t)-T_{q}\right)$

in which $T_{q}[C]$ is the temperature of the bulk quenching liquid in turbulent flow, and $h(t)\left[\mathrm{J} \cdot \mathrm{s}^{-1} \cdot \mathrm{m}^{-1} \cdot \mathrm{K}^{-1}\right]$ is the heat transfer coefficient of the boundary layer of the liquid at the surface of the probe. Besides the boundary condition equation (2) must be completed with the initial conditions that have to be compatible with the boundary conditions, too. This compatibility was guaranteed as follows: to solve the boundary conditions in (5) a refreshed value $T$ in grid point $r_{N-1}$ was estimated by using the $1^{\text {st }}$ backward spatial derivative of the already refreshed points as in (6)

$T\left(r_{N-1}, t\right) \approx T\left(r_{N-2}, t\right)+$

$$
\frac{\left(r_{N-1}-r_{N-2}\right)\left(T\left(r_{N-2}, t\right)-T\left(r_{N-3}, t\right)\right)}{\left(r_{N-2}-r_{N-3}\right)}
$$

and $T\left(r_{N}, t\right)$ was estimated by the use of the heat transfer coefficient as in (7)

$T\left(r_{N}, t\right) \approx \frac{h\left(T\left(r_{N-1}, t\right)\right) T_{q}+k\left(T\left(r_{N-1}, t\right)\right) / \Delta r}{h\left(T\left(r_{N-1}, t\right)\right)+k\left(T\left(r_{N-1}, t\right)\right) / \Delta r}$ 
To make the above construction practically useful the number of the grid-points $N$ and the step-length of the Euler-integration according to the time, $\delta t$, must be determined. This question is critical due to the singularity in (2) at $r=0$. For this purpose the "trial and error" method was chosen: the cooling curves were calculated for Planck's model in (1a) for the pairs $\left\{N=20, \delta t=10^{-2}[s]\right\}$, and $\left\{N=100, \delta t=10^{-3}[s]\right\}$, and the graphs were plotted in the same charts in Fig. 1. It reveals that for the calculations it is sufficient to use the coarser one that considerably reduces the computational burden and time.

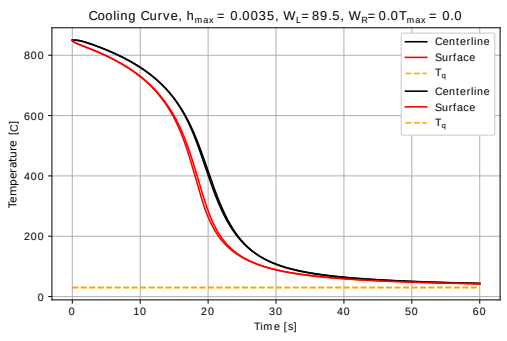

a)

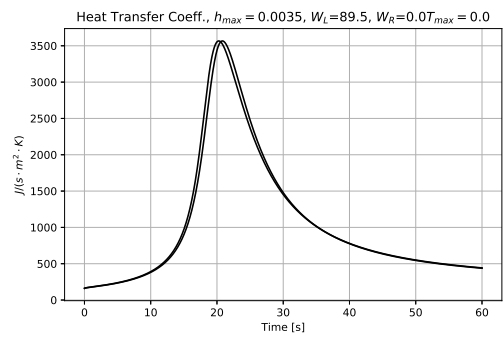

b)

Figure 1

The cooling curves (a) and the HTC values for Planck's model in (1a) for the pairs $\{N=20, \delta t=$ $\left.10^{-2}[s]\right\}$, and $\left\{N=100, \delta t=10^{-3}[s]\right\}$ (b)

\section{The basic optimization algorithm}

In the suggested approach, to represent the "target cooling curve", one of the "format functions" in (1) can be selected with the parameter values given in Table 1. Then, by applying the FEM method described in Section 2, for the points of the discrete time-grid applied, the cooling curve $T_{c} \in \mathbb{R}^{L}, L \in \mathbb{N}$ as an array can be computed. Following that the error of the difference of the arrays defined as $E \stackrel{\text { def }}{=}\left\|T_{c}-T_{\text {target }}\right\|^{v}$ can be computed. Finally, the scalar function $E(x) \equiv E\left(h_{\max }, T_{\max }, w_{\text {left }}, w_{\text {right }}\right): \mathbb{R}^{4} \mapsto \mathbb{R}$ can be minimized by varying its 4 independent arguments. Normally, in the practice using quadratic cost functions (i.e. $v=2$ in the Frobenius norm) happens because of rather "technical" than "mathematical" reasons. In the present simulation it was found that the choice of $v=0.25$ produces a "convenient" cost function. If the local optimum must be approached under constraints, Lagrange's "Reduced Gradient Method" can be applied for a given initial argument $x_{0}$. If there are no constraints, the Gradient Descent method generates a sequence of approximations as $x_{i+1}=x_{i}+\alpha_{i} \nabla E_{i}, i \in\{0,1,2, \ldots\}$ in which $\alpha_{i}$ is a small negative number if the aim is to minimize $E(x)$. If no a priori information is available on $\|\nabla E(x)\|$, very small $\alpha_{i}$ must be chosen. If some assumption is available for the minimum, a big step can be done in the direction of the gradient that could approach this minimum in a single step, then the same consideration can be repeated until reaching this minimum "Newton-Raphson Algorithm". Since this method converges well only if the available minimum can be precisely estimated in advance, we have the freedom to choose various $\alpha_{i}$ values. 
The idea of accelerating the Newton-Raphson Algorithm consists of the combination of various solutions borrowed from already known methods as follows: i) From the Simplex Algorithm the idea of shrinking the simplex in the vicinity of the local optimum is borrowed. The speed of the motion of the simplex roughly is proportional to its size. It can quickly approach the local minimum but its drift stops in its vicinity. After shrinking, the smaller simplex drifts with reduced speed but finds the optimum with better precision. For this purpose a "Shrinking Parameter" $v \in(0,1)$ is introduced as $\alpha \Leftarrow v \cdot \alpha$. ii) From the Particle Swarm Optimization the idea of storing the values of the already found best solution is borrowed. iii) Similar problem may happen when the shrinking operation occurs too frequently. This means the appearance of very small factors containing $v^{k} \rightarrow 0$ as $\mathbb{N} \ni k \rightarrow \infty$, therefore the algorithm may become very slow. In the calculations $v=0.6$ was chosen.

The Newton-Raphson algorithm has alternatives as e.g. the "Fixed Point Iteration"based methods that may work well, too. Their essence is Banach's Fixed Point Theorem [11] according to which the solution of certain numerical problems can be reformulated as finding the fixed point of a contractive map defined over a complete linear normed metric space. This fixed point subsequently can be approached by a simple iteration as follows. Consider the (generally nonlinear) real function $f: \mathbb{R}^{n} \mapsto$ $\mathbb{R}^{n}, n \in \mathbb{N}$ with the mathematical problem $q^{\text {Des }}=f\left(q_{\star}\right)$ in which $q^{\text {Des }}$ is a known output (the "response") for which we have to find the appropriate input $q_{\star}$. Dineva in [12] suggested the iteration for finding the appropriate input defined as a sequence of deformed inputs as:

$q^{D e f}(i+1)=\left[F\left(A_{c}\|h(i)\|+x_{*}\right)-x_{*}\right] e(i)+q^{D e f}(i)$

with the "response error" defined as $h(i) \stackrel{\text { def }}{=} f\left(q^{\text {Def }}(i)\right)-q^{\text {Des }}(i+1)$, and the vector of unit Frobenius norm $e(i) \stackrel{\text { def }}{=} \frac{h(i)}{\|h(i)\|}$. Here $A_{c} \in \mathbb{R}$ is the adaptive control parameter, and $F: \mathbb{R} \mapsto \mathbb{R}$ is a real differentiable function with an attractive fixed point $F\left(x_{\star}\right)=x_{\star}$. Evidently, if $h(i)=0$ then $q^{\operatorname{Def}}(i+1)=q^{\operatorname{Def}}(i)$, that is the solution of the control task is the fixed point of this problem. By considering the $1^{\text {st }}$ order Taylor series approximation of $F(x)$ around $x_{\star}$ and $f\left(q^{D e f}\right)$ around $q_{\star}$ Dineva proved that an appropriate $A_{C}$ can be chosen for obtaining a convergent sequence if the real part of each eigenvalue of $\left.\frac{\partial f}{\partial q^{D e f}}\right|_{\ddot{q}_{\star}} \in \mathbb{R}^{n \times n}$ is either positive or negative. In (8) various $F(x)$ functions can be chosen. Instead of choosing some analytical formula in [13] a special function was applied that can be realized and well configured in a program block. It transforms the vector $b \in \mathbb{R}^{n}$ into the vector $a \in \mathbb{R}^{n}(\|a\| \neq\|b\|)$ via so augmenting them with a physically not interpreted $(n+1)^{\text {th }}$ dimension that the augmented vectors have the same Frobenius norm. Then an orthogonal matrix is analytically computed that rotates the augmented variant of $\mathbf{b}$ into that of $\mathbf{a}$ while leaves their orthogonal subspace invariant. With an interpolation parameter $\lambda \in(0,1)$ the angle of the full rotation can be multiplied, and this "moderated rotation" can be applied. The projection of the rotated augmented vector in the original space suffers simultaneous rotation and shrinking/dilatation as it approaches vector a. This idea evidently can be applied for $\nabla E(x)$ that can be driven to zero by fixed point iteration if in the place of the desired 
value $q^{\text {Des }} \equiv 0$, in the role of $q^{\text {Def }}$ the variable $x$, and in the role of the realized value $f\left(q^{D e f}\right)$ the quantity $\nabla E(x)$ are written: $x_{n+1}=\Phi\left(\nabla E_{n}, x_{n}, 0\right)$. Roughly speaking, in the vicinity of the local minimum, by driving $\nabla E(x)$ to zero by a simple fixed point iteration can be applied. In the lack of information whether the actual point is in the basin of attraction of a local minimum or a local maximum (we wish to evade the calculation of $J:=\frac{\partial^{2} E}{\partial x_{i} \partial x_{j}}$ ), this algorithm may proceed toward a local maximum while its counterpart based on the Gradient Descent approach always moves towards the local minimum. The speed of its migration also depends on the Jacobian $J$. However, the behaviour of the fixed point iteration can be better and can be worse than that of its gradient descent counterpart. To tackle this problem the following procedure was applied: the Fixed Point Iteration was modified as follows: to evade too big jumps, in the goal for the step $(i+1)$ instead of 0 the reduced variant of the previous value $\kappa E_{i}$ has been written with $\kappa \in(0,1)$. In this manner a finite value slowly and cautiously can be driven toward 0 . To speed up the convergence, the next point in the space of the independent variables was selected as

$$
\begin{aligned}
& x_{n+1}=\Phi\left(\nabla E_{n}, x_{n}+\mu_{n}\left(x_{n}-x_{n-1}\right), \kappa \nabla E_{n}\right), \\
& \mu_{n}=\frac{\omega_{4} \tanh \left(1 /\left(\omega_{3}+\widetilde{\|J\|}\right)\right)}{\tanh \left(1 / \omega_{3}\right)}
\end{aligned}
$$

in which $\widetilde{\|J\|}$ is a roughly estimated, filtered "Jacobian". Regarding the filter, by introducing a "smoothing and forgetting factor" $\eta \in(0,1)$, and utilizing that $\sum_{\ell=0}^{\infty} \eta^{\ell}=$ $1 /(1-\eta)$, any discrete time-dependent quantity $f\left(t_{i}\right)$ can be replaced with its filtered value $\tilde{f}\left(t_{i}\right)=(1-\eta) \sum_{\ell=0}^{\infty} \eta^{\ell} f\left(t_{i-\ell}\right)$. This corresponds to the weighted average of the recent values of $f$ in which the very old contributions are gradually forgotten due to their small weighting coefficients. In the calculations $\eta=0.9$ was applied. Evidently, if $\widetilde{\|J\|} \ll \omega_{3}$, then $\mu_{n} \approx \omega_{4}$, that means that for very slow process the "accelerator" factor has some limit. For $\widetilde{\|J\|} \rightarrow \infty \mu_{n} \rightarrow 0$, i.e. if the process is fast enough, practically no process acceleration happens. The simple approximation in (10) was applied.

$\widetilde{\|J\|_{n}}=\frac{\left\|\nabla E_{n} \widetilde{-\nabla E_{n-1}}\right\|}{\left\|\widetilde{x}_{n}-x_{n-1}\right\|+\omega_{2}}$

in which $\omega_{2}>0$ has the role of evading division by zero. The computation of (10) evidently means far less burden than the numerical estimation of the real Jacobian. However, it does not contain information on the direction of the drift of $x$, so it can be quite unreliable, and may not result in monotonic decrease of $E$. However, it sooner may produce better values than the Newton-Raphson algorithm, and this better value can be stored and used even if the estimation based on (10) later diverges. In the computations $\kappa=0.25, \omega_{4}=1.0, \omega_{3}=3.0, \omega_{2}=10^{-6}, \tilde{R}=10^{2}$, and $\lambda=0.1$ were used. For 5 steps the Newton-Raphson method run to create the "antecedents" for the fixed point iteration, then the computations turned to it. In Section 4 typical numerical results are provided. 


\section{Numerical computations}

The time need of the computations depends on the hardware and software applied. The computations were made on a Dell inspiron 15R laptop operated by the central processor Intel ${ }^{\circledR}$ Core $^{\mathrm{TM}}$ i5-3337U CPU @ $1.80 \mathrm{GHz} \times 4$ under Ubuntu ver. 13.04 operating system without using graphical acceleration. The sequential program was written in Julia Version 1.0.3 (2018-12-18). This program language is developed at the MIT, it is a free software, it is very similar to the MATLAB, but it runs almost as fast as a $C$ code [14]. It provides its users with a standard macro that measures the time of computing the value of a function. It was experimentally found that for the calculation of $E$ and $\nabla E$ approximately $0.9-1.2 / s$ was used, the abstract rotations were calculated during $45-90 / \mu s$. During the research 8 different scenarios were investigated. In each of them a Newton-Raphson algorithm and a FPI-based solution were compared according to the already given parameter settings. In the first group the cases with possible exactly 0 approximation error (each "target" was created by the same format function using different starting parameters) were considered. It was found that the occurrence of the exponential function in the definitions (1a) and (1b) needed quite slow and cautious approximation procedure, therefore their use was less successful. However, the present settings was successful for the approximation of the functions in (1c) and (1d) that do not contain the exponential function. In Fig. 2 the target in (1d) was approximated with the initial element (1d). In this case the FPI-based approach very early provided the best solution.

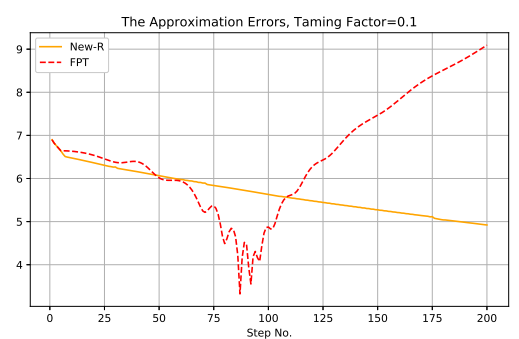

a)

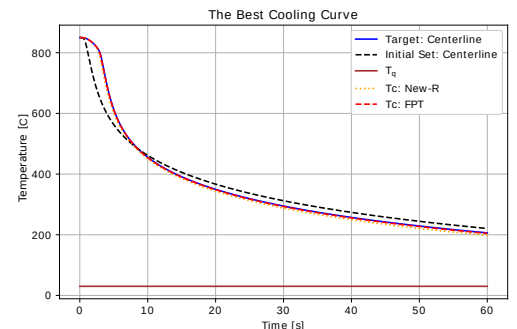

c)

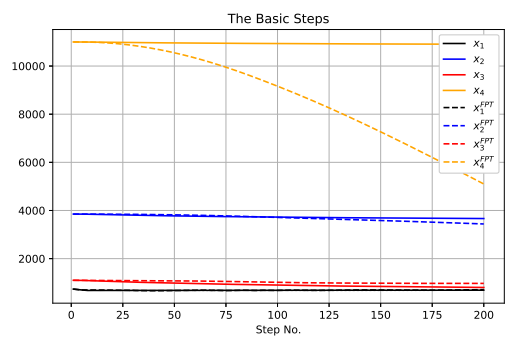

b)

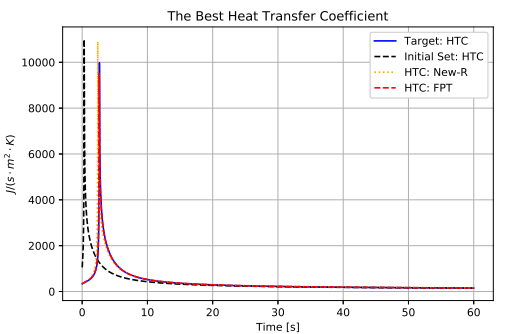

d)

Figure 2

The cost function (Error) (a), the tuned variables $x=\left[T_{\max }, w_{\text {left }}, w_{\text {right }}, h_{\max }\right](\mathrm{b})$, the cooling curve (c), the heat transfer coefficient HTC (d) 
In the following runs variants in (1c) and (1d) as initial HTC distributions were used to approximate targets created by some different distribution. When the target (1c) was approximated by the form function (1d) the Newton-Raphson method provided the better approximation. The reversed problem, i.e. when the target (1d) was approximated by the form function (1c) the FPI-based solution was better but the Newton-Raphson-based approach resulted in quite good approximation, too. Figure 3 corresponds to the approximation of (1a) by the function (1c). In this case the FPI-based approach yielded a surprisingly good result.

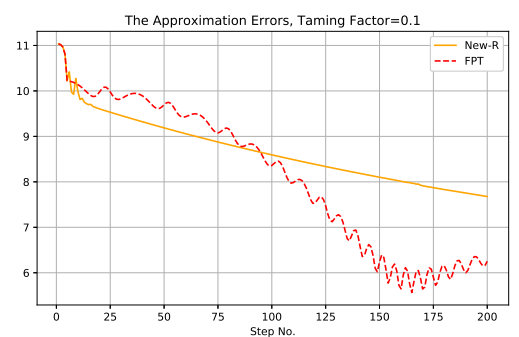

a)

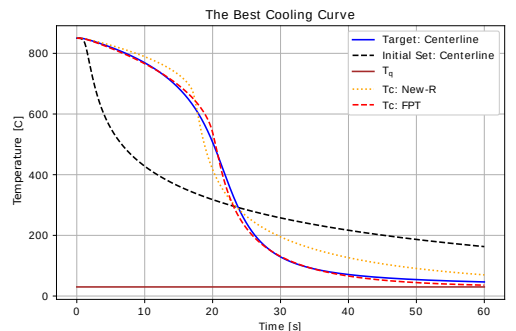

c)

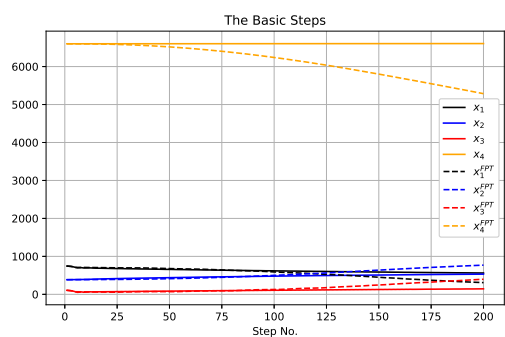

b)

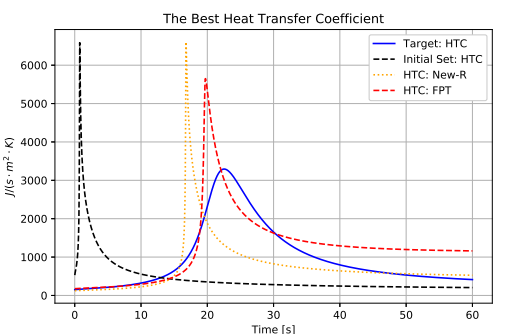

d)

Figure 3

The cost function (Error) (a), the tuned variables $x=\left[T_{\max }, w_{\text {left }}, w_{\text {right }}, h_{\max }\right](\mathrm{b})$, the cooling curve (c), the heat transfer coefficient HTC (d)

\section{Conclusion}

In this paper a simple computational method was suggested for numerically tackling the inverse heat conduction problem that has great significance in metallurgy. By utilizing the geometric and thermodynamic simplifications offered by the standard ISO 9950 a single dimensional space $r$ and the time $t$ variables were considered by using the thermal data of the alloy Inconel 600. The finite elements approach, dealing with the singularity in the centre of the polar coordinates, the boundary conditions and the initial conditions that must be compatible with the boundary conditions were discussed in details. For setting the appropriate grid-points in space and time the method of "trial and error" was applied. A novel solution was outlined that works with an "entity" that evolves according to the Newton-Raphson Algorithm with step-by-step reduction of its step length, and also computes the propagation of an associated "partner entity" that evolves according to a fixed point iteration. While 
it can be taken for granted that the original "entity" converges to a local optima but its speed of convergence becomes very slow, the "partner entity" may have very fast convergence but it may diverge, too. Numerical simulations were elaborated for the combination of four typical "format functions" that can capture the essential asymmetry in the $\operatorname{HTC}(T)$ function, and numerically can approach the measured data taken from the literature. Regarding the computational burden, the requirements of both approaches are comparable. The computations were realized by a freely available program language (Julia) that almost as efficiently utilizes the available hardware as a $\mathrm{C}$ code. No any special FEM software was applied. While the computation of the gradient of the cost function needed the time between $0.9[s]$ and $1.2[s]$, the time need of the calculation of the abstract rotation applied by the FPI-based solution was between $45[\mu s]$ and $85[\mu s]$, i.e. it was negligible in comparison with the computational needs of the error gradient.

The preliminary results indicate that there are more or less plausible possibilities for further speeding up the recommended approach as follows: a) instead of the complete array taken from the time grid under consideration more sparse samples can be used for the calculation of the error function that has to be minimized; b) instead applying more dense points in the vicinity of the important "segments" of the target cooling curve, enhanced weighting of the contribution of these points in the error function may be practical, too; c) due to the special shape of a particular cooling curve the success of approximation strongly depends on the starting parameters of the approximation; it seems to be practical to simultaneously run several "associated couples" pairs, and finally select the best result. Since the considered approximations needed very limited running time on a "common" hardware, it can be concluded that it will be expedient to conduct further modelling investigations. The present approximation is so simple that it seems to be useful in the education, too.

\section{Acknowledgement}

The financial support of this work by Hungarian-Mexican bilateral project (20192.1.11-TÉT-2019-00007) is acknowledged.

\section{References}

[1] ISO9950, ISO 9950: Industrial Quenching Oils - Determination of Cooling Characteristics Nickel-Alloy Probe Test Method 1995(E). ISO, Switzerland, 1995.

[2] J. Clark and R. Tye, "Thermophysical properties reference data for some key engineering alloys," High Temperatures - High Pressures, vol. 35-36, pp. 1$14,2003-2004$.

[3] D. Landek, J. Župan, and T. Filetin, "A prediction of quenching parameters using inverse analysis," Materials Performance and Characterization, vol. 3, no. 2, pp. 229-241, 2014. 
[4] S. Szénási and I. Felde, "Configuring genetic algorithm to solve the inverse heat conduction problem," ACTA POLYTECHNICA HUNGARICA, vol. 14, no. 6, pp. 133-152, 2017.

[5] L. Zadeh, "Fuzzy sets," Information and Control, vol. 8, pp. 338-353, 1965.

[6] J. Dombi, "A general class of fuzzy operators, the de Morgan class of fuzzy operators and fuzziness measures induced by fuzzy operators," Fuzzy Sets and Systems, vol. 8, pp. 197-216, 1982.

[7] J. Dombi, T. Jónás, and Z. Tóth, "The epsilon probability distribution and its application in reliability theory," Acta Polytechnica Hungarica, vol. 15, no. 1, pp. 197-216, 2018.

[8] J. Tenreiro Machado and V. Kiryakova, "The chronicles of fractional calculus," Fract. Calc. Appl. Anal., vol. 20, no. 2, pp. 307-336, 2017.

[9] I. Felde, "Liquid quenchant database: determination of heat transfer coefficient during quenching," Int. J. Microstructure and Materials Properties, vol. 11, no. 3/4, pp. 277-287, 2016.

[10] R. Mastrullo, A. Mauro, A. Rosato, and G. Vanoli, "Comparison of R744 and $\mathrm{R} 134 \mathrm{a}$ heat transfer coefficients during flow boiling in a horizontal circular smooth tube," Proc. of the International Conference on Renewable Energies and Power Quality (ICREPQ'09), 15th to 17th April, 2009, Valencia, Spain, vol. 1, no. 7, pp. 577-581, 2009.

[11] S. Banach, "Sur les opérations dans les ensembles abstraits et leur application aux équations intégrales (About the Operations in the Abstract Sets and Their Application to Integral Equations)," Fund. Math., vol. 3, pp. 133-181, 1922.

[12] A. Dineva, J. Tar, and A. Várkonyi-Kóczy, "Novel generation of Fixed Point Transformation for the adaptive control of a nonlinear neuron model," In proc. of the IEEE International Conference on Systems, Man, and Cybernetics, October 10-13, 2015, Hong Kong (SMC 2015), pp. 987-992, 2015.

[13] B. Csanádi, P. Galambos, J. Tar, G. Györök, and A. Serester, "A novel, abstract rotation-based fixed point transformation in adaptive control," In the Proc. of the 2018 IEEE International Conference on Systems, Man, and Cybernetics (SMC2018), October 7-10, 2018, Miyazaki, Japan, pp. 2577-2582, 2018.

[14] J. Bezanson, A. Edelman, S. Karpinski, and V. B. Shah, "Julia," https://julialang.org, 2019.

[15] S. Szénási, "Solving the inverse heat conduction problem using NVLink capable power architecture," PeerJ Computer Science, vol. 3, p. e138, nov 2017. 